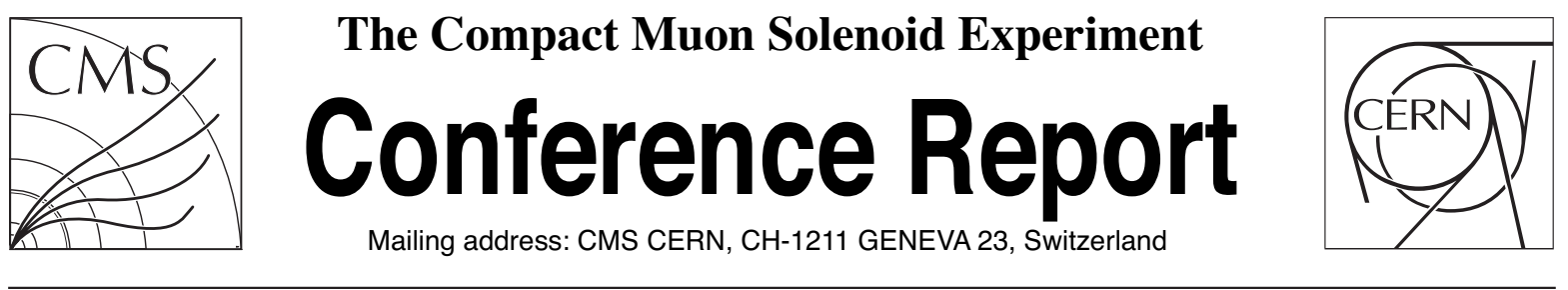

07 February 2020 (v6, 09 August 2021)

\title{
The Silicon Sensors for the High Granularity Calorimeter of CMS
}

Peter Paulitsch, on behalf of the CMS Collaboration

\begin{abstract}
The installation of the High-Luminosity Large Hadron Collider (HL-LHC) presents unprecedented challenges to experiments like the Compact Muon Solenoid (CMS) in terms of event rate, integrated luminosity and therefore radiation exposures. To cope with this new environment, new detectors will be installed during the CMS Phase 2 Upgrade, including the replacement of the calorimeter endcaps with the "High Granularity Calorimeter" (HGCAL), which contains silicon sensors and scintillators as active elements. The silicon sensors will be produced in an 8" wafer process, which is new for high-energy physics, so it demands extensive quality verification. A first batch of prototype sensors underwent electrical tests at the institutes of the CMS Collaboration. Testing revealed major problems with the mechanical stability of the thin backside protective layer, that were not seen in earlier 6" prototypes produced by a different backside processing method. Following these results, the HGCAL group introduced the concept of "frontside biasing", allowing testing of the sensors without exposing its backside, verified the applicability, and adapted the prototype design to apply this method in series production.
\end{abstract}

Presented at HSTD12 12th International Hiroshima Symposium on the Development and Application of Semiconductor Tracking Detectors,

Published in Nuclear Inst. and Methods in Physics Research A, doi: https://doi.org/10.1016/j.nima.2020.164428 


\title{
The silicon sensors for the High Granularity Calorimeter of CMS
}

\author{
Peter Paulitsch, on behalf of the CMS Collaboration \\ Austrian Academy of Sciences, Institute of High Energy Physics (HEPHY), Nikolsdorfer Gasse 18, 1050 Wien, Austria
}

\section{A R T I C L E I N F O}

\section{Keywords:}

Compact muon solenoid

Large hadron collider

High-luminosity

High Granularity Calorimeter

Large area

Silicon pad sensors

\begin{abstract}
A B S T R A C T
The installation of the High-Luminosity Large Hadron Collider (HL-LHC) presents unprecedented challenges to experiments like the Compact Muon Solenoid (CMS) in terms of event rate, integrated luminosity and therefore radiation exposures. To cope with this new environment, new detectors will be installed during the CMS Phase 2 Upgrade, including the replacement of the calorimeter endcaps with the "High Granularity Calorimeter" (HGCAL), which contains silicon sensors and scintillators as active elements. The silicon sensors will be produced in an 8" wafer process, which is new for high-energy physics, so it demands extensive quality verification. A first batch of prototype sensors underwent electrical tests at the institutes of the CMS Collaboration. Testing revealed major problems with the mechanical stability of the thin backside protective layer, that were not seen in earlier 6" prototypes produced by a different backside processing method. Following these results, the HGCAL group introduced the concept of "frontside biasing", allowing testing of the sensors without exposing its backside, verified the applicability, and adapted the prototype design to apply this method in series production.
\end{abstract}

\section{Introduction}

During the Phase-2 Upgrade (2025 to 2027), the Large Hadron Collider (LHC) will be upgraded to the High-Luminosity LHC (HL-LHC) [1]. The HL-LHC will have a factor 5-7 higher instantaneous luminosity compared to the end of LHC operation, resulting in a proportionally higher pileup and a factor 10 increase in integrated luminosity $\left(3000 \mathrm{fb}^{-1}\right)$ over 10 years of operation. As a result, unprecedented levels of radiation and particle shower densities will affect experiments such as the Compact Muon Solenoid (CMS) [2]. At these high collision rates, the overlap of particle showers will not be negligible any more, so detectors with increased spatial resolution are needed to distinct different showers. To address these challenges, the CMS Collaboration will upgrade its subdetectors including a replacement for the existing endcap calorimeters with the new High Granularity Calorimeter (HGCAL) [3], as shown in Figs. 1 and 2. The calorimeter will utilize about $30000^{1}$ sensor modules covering more than $620 \mathrm{~m}^{2}$, allowing for efficient mitigation of pileup and particle-flow calorimetry.

The HGCAL will be a sandwich calorimeter and will include an electromagnetic part (Calorimeter Endcap - Electromagnetic, CE-E) and a hadronic part (Calorimeter Endcap - Hadronic, CE-H). While the active sensing elements of the electromagnetic part will be entirely made of silicon sensors, the hadronic elements will implement silicon just for the inner high radiation domain. At the outer regions with lower radiation levels, plastic scintillators coupled to silicon photomultipliers [3] will be used, as shown in Fig. 2. This article focuses on the silicon parts of the CE-E and CE-H sections.

\section{Silicon sensors for the HGCAL}

The silicon sensors of the HGCAL will be produced in an $8^{\prime \prime}$ process [3], in contrast to earlier applications in high-energy physics, which used 6 " processes. The leap towards the $8^{\prime \prime}$ process decisively reduces production costs and sensor testing efforts. However, this process is new to large-area sensors for high-energy physics and therefore brings new challenges in terms of radiation hardness, high-voltage stability, and other issues like sensor backside sensitivity (see Section 3.2). Three different sensor thicknesses will be deployed: $120 \mu \mathrm{m}, 200 \mu \mathrm{m}$ and $300 \mu \mathrm{m}$. Radiation damage increases the sensor leakage current. Thinner sensors draw less leakage current, so these will be utilized in regions with increased radiation levels to cope with higher radiation damage induced leakage currents (Table 1).

Hamamatsu will manufacture the $120 \mu \mathrm{m}$ sensors in an epitaxial process, whereas the thicker will be produced in a float-zone process, as shown in Fig. 3.

The shape of a full sensor is hexagonal (see Fig. 4) because a hexagon is the largest seamlessly tileable, regular shape on a circular wafer. This maximizes the wafer-area usage, reduces the number of necessary sensor tiles, hence decreases costs.

Consequently, the active sensing elements also have a hexagonal shape, except for some irregular cells at the sensor edges and corners. Studies from $\mathrm{RD}^{2} 0^{2}$ [7] have shown that $\mathrm{n}$-in-p materials have better charge collection than $\mathrm{p}-\mathrm{in}-\mathrm{n}$, so like in the CMS Tracker [8], n-in-p

E-mail address: peter.paulitsch@cern.ch.

1 This value is preliminary, it may be changed for optimized calorimeter coverage by partial sensors, see Section 3.3

2 Radiation hard semiconductor devices for very high luminosity colliders, http://rd50.web.cern.ch/rd50/. 


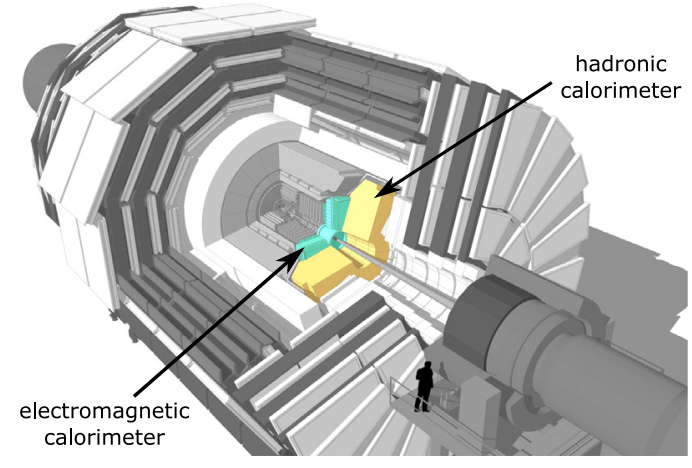

Fig. 1. Location of the HGCAL at the CMS endcaps [4].

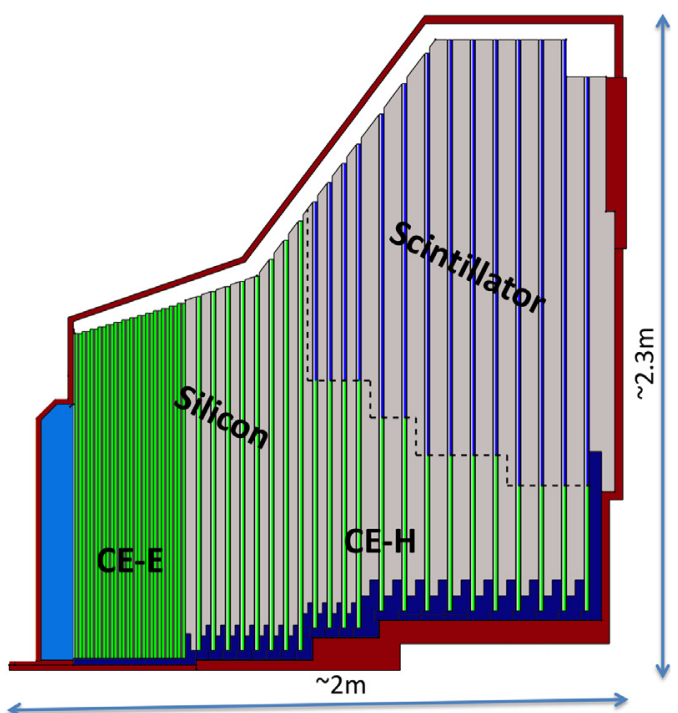

Electromagnetic calorimeter (CE-E):

$\mathrm{Si}, \mathrm{Cu} \&$ CuW \& Pb absorbers, 28 layers, $25 \mathrm{X}_{0} \& \sim 1.3 \lambda$ Hadronic calorimeter (CE-H):

Si \& scintillator, steel absorbers, 22 layers, $\sim 8.5 \lambda$

Fig. 2. Schematic cross section of an endcap sector [5].

Table 1

Active thicknesses $d_{\text {act }}$, number of channels, maximum expected fluences $\Phi_{\text {neq }}$ (normalized to $1 \mathrm{MeV}$ neutron equivalent), and maximum expected total ionizing dose (TID) at $3000 \mathrm{fb}^{-1}$ [3]. $\Phi_{\text {neq }}$ is defined as the number of neutrons passing per sensor area and typically describes lattice displacement damage. The energy spectrum is normalized to $1 \mathrm{MeV}$ monoenergetic neutrons. The TID is the dose by charged particles, and typically describes damages in oxide layers, caused by generating and accumulating immobile charges.

\begin{tabular}{llll}
\hline$d_{\text {act }}(\mu \mathrm{m})$ & Full-size channels & $\Phi_{\text {neq }}\left(\mathrm{cm}^{-2}\right)$ & TID (Gy) \\
\hline 120 & $432(\mathrm{HD})$ & $7.0 \times 10^{15}$ & $1 \times 10^{6}$ \\
200 & $192(\mathrm{LD})$ & $2.5 \times 10^{15}$ & $2 \times 10^{5}$ \\
300 & $192(\mathrm{LD})$ & $5.0 \times 10^{14}$ & $3 \times 10^{4}$ \\
\hline
\end{tabular}

diodes were chosen also for the HGCAL. For the $200 \mu \mathrm{m}$ and $300 \mu \mathrm{m}$ thick sensors, the so-called "Low-Density" (LD) design with 192 full-size channels is chosen. To keep the diode (cell) capacitances and therefor series noise in the preamplifier low, the $120 \mu \mathrm{m}$ thick sensors have smaller cells and therefore 432 full-size channels, the "High-Density" (HD) design as shown in Fig. 4. To control the electric fields and to reduce the leakage current at the edge region, the sensor features two $\mathrm{n}^{++}$doped guard rings; the inner one is connected to ground and the outer one is floating. A $p^{++}$doped edge ring is placed at the sensor edge, as shown in Fig. 11. This edge ring brings the edge region to the same potential as the $p^{++}$doped backside implant. Otherwise, microscopic

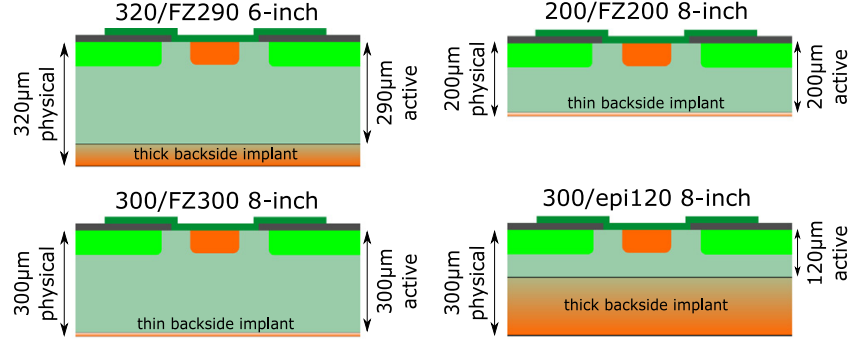

Fig. 3. Sensor cross sections at different manufacturing processes. "epi" denotes an epitaxial process, "FZ" stands for "float-zone". For comparison, one 6" processed sensor cross section is shown. The thin backside implant of the FZ200 and FZ300 processes is about $1 \mu \mathrm{m}$ thick.

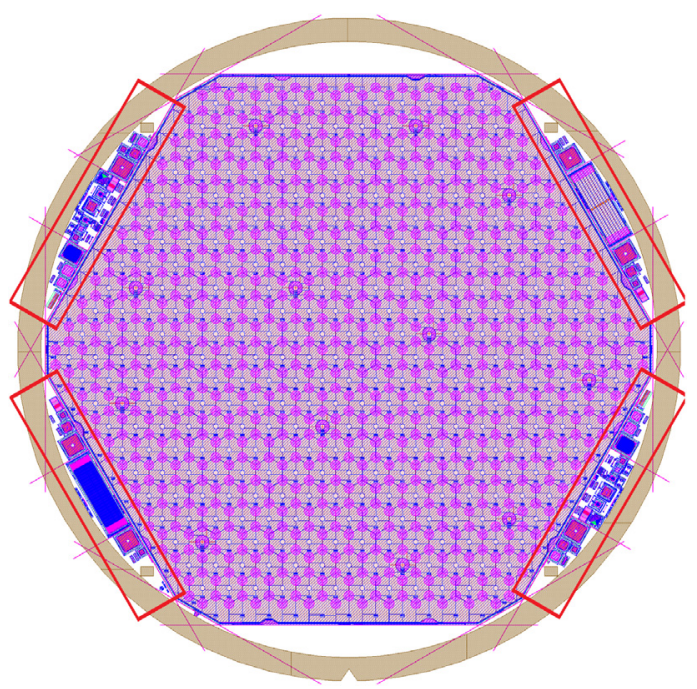

Fig. 4. 432-channel (HD) full wafer design, thickness $120 \mu \mathrm{m}$. The red squares at the wafer periphery mark the four test structure half moons [6].

cracks at the cutting edges would lead to field spikes which may cause breakdowns.

\section{Sensor testing}

\subsection{Test system}

During the production phase, multiple CMS institutes will do quality control of $1-2 \%$ of all produced sensors. Because of the large quantities of sensors produced, CMS needs fast and widely automatized test equipment. Probe-cards are a common standard in the semiconductor industry for large-scale quality control because they allow fast and easily reproducible measurements. Therefore the collaboration developed an open-source full wafer probe-card and switching system, called "ARRAY" [9]. A probe-card consists of a carrier which holds a set of probe tips in a defined geometric layout.

Characterization of the sensors is primarily fulfilled by measuring current versus voltage (IV) and capacitance versus voltage (CV) curves [10,11]. For all individual sensor cells on the same wafer, these measurements allow extracting parameters like the full depletion voltage $\left(V_{\mathrm{fd}}\right)$, full depletion capacitance $\left(C_{\mathrm{fd}}\right)$, or the breakdown voltage $\left(V_{\mathrm{bd}}\right)$.

\subsection{Sensor backside sensitivity and scratch tests}

Diode current characteristics on 8 " HGCAL sensor prototypes showed a degradation in terms of an increasing number of cells with 


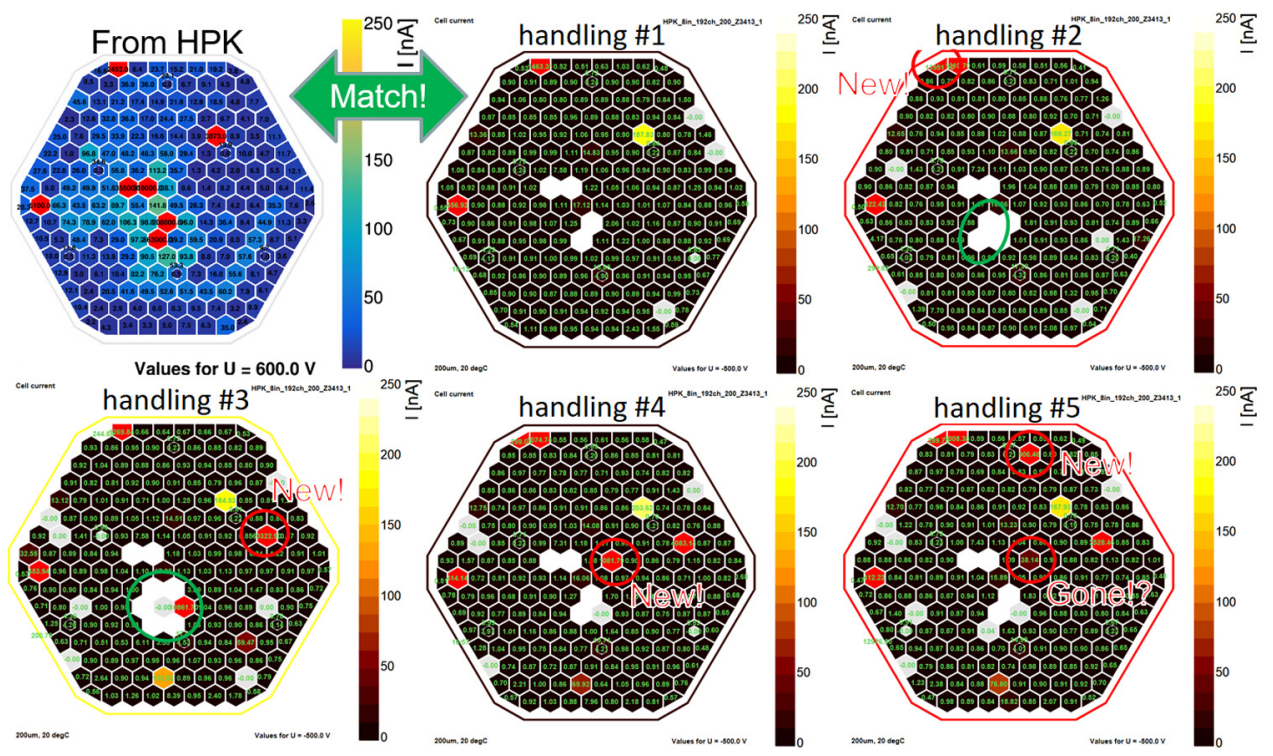

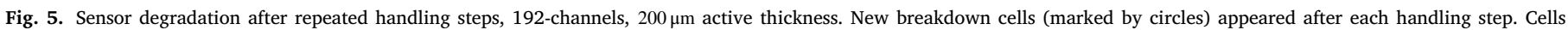
with values of " 0.00 " have bad contacts. The first test ("From HPK") was done by Hamamatsu before shipment.

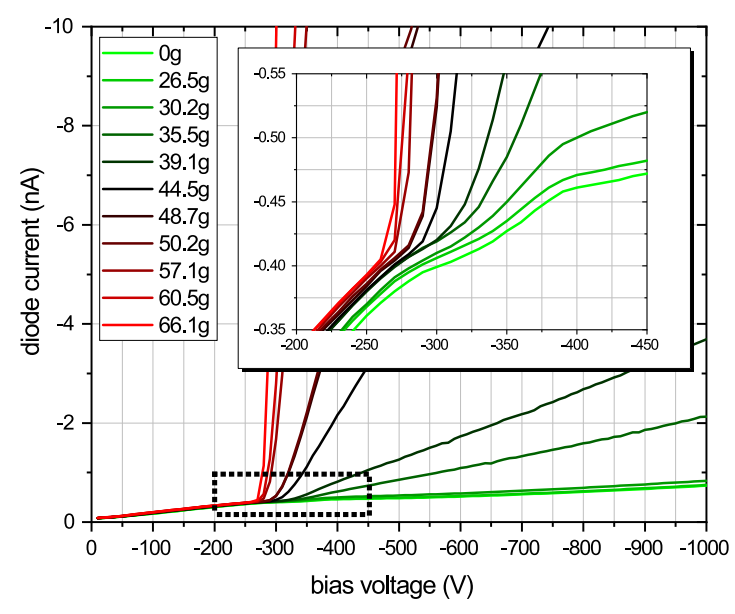

Fig. 6. Test structure diode currents as a function of the bias voltage for increasing scratch depth, indicated by needle weight. The zoomed inlay shows a reduction of the breakdown voltage and increased currents with higher needle weight.

early breakdowns as shown in Fig. 5. This degradation only occurred after sensor handling procedures were done. Repeated measurements without handling procedures in between remained stable. Because earlier $6^{\prime \prime}$ prototypes did not show this behavior, we suspected that the thin backside metalization of the $8^{\prime \prime}$ prototypes might cause early breakdowns and increased sensor currents. The previous $6^{\prime \prime}$ prototypes had a backside with a thick ("deep-diffused") field stop implant, seen on Fig. 3. The purpose of the highly doped field stop is to have zero electrical field at the beginning of the backside metalization. This stops the spread of the field also at high bias voltage.

To investigate these problems with the fragile backside, scratch tests with a tungsten carbide needle were performed on test structures [12, 13]. Using a needle manipulator, the needle tip ( $50 \mu \mathrm{m}$ diameter) was kept hovering over the test structure diode, which was fixed to a precision scale (Kern 572-30, reproducibility $1 \mu \mathrm{g}$ ). The underlying table was raised until the needle touched the sensor surface and the scale displayed the desired weight. Subsequently, the table was moved to create a scratch. After each scratch, the needle was removed and the diode IV characteristics were recorded, as shown in Fig. 6. These tests showed that the diode's backside is highly sensitive to scratches,

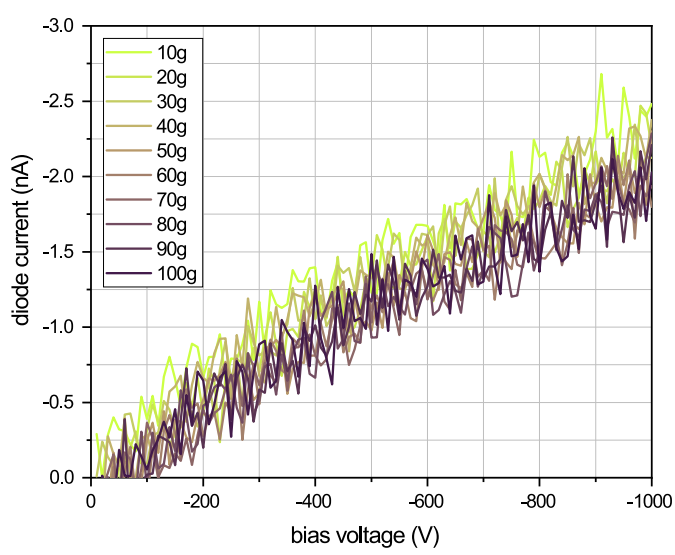

Fig. 7. Test structure diode currents as a function of the bias voltage for increasing pin pressure, indicated by pin weight. No systematic behavior is seen at different pin pressures.

the breakdown voltage decreased with scratches made at higher needle weights and diode currents increased at deeper scratches. Depth measurements via laser interferometry (Wyko NT 3300 Profiling System) of these scratches showed that indeed the scratch depth increases with higher needle weight, and that all tried weights (10 $\mathrm{g}$ and more) penetrated the $1.1 \mu \mathrm{m}$ backside aluminum layer.

Vertical pressure tests with variable pressures of the probe-card pins showed that the applied pressure of these pins did not alter the electrical characteristics of the diodes, even when measured during applied pressure, as shown in Fig. 7.

\subsection{Frontside biasing and edge/guard ring extensions}

Initially, it was planned to apply the bias voltage during testing via the sensor's backside aluminum metalization. Due to the aforementioned backside fragility, Hamamatsu discussed with the collaboration to glue a compound polyimide (Kapton ${ }^{\mathrm{TM}}$ ) foil on the backside during production to prevent scratches. This procedure would make it impossible to directly contact the backside. By exploiting the lowresistance path between edge ring, bulk and backside implant (all are p-doped, shown in Fig. 8), it is possible to use the edge ring as a 


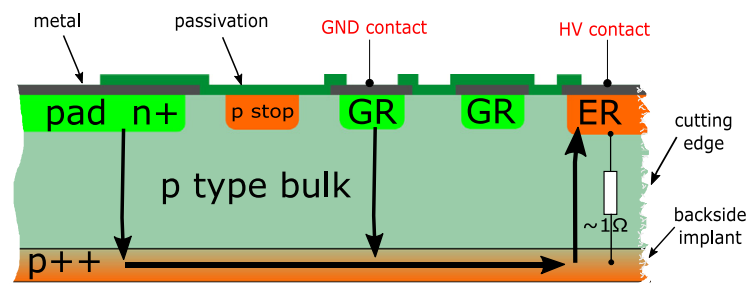

Fig. 8. Sketch of cross section of the sensor edge using the frontside biasing concept. Current flow is visualized by the black arrows. Since there is a low resistance $(\sim 1 \Omega)$ path between edge ring and backside implant, the current flows via the backside to the edge ring, where the HV contact is applied.

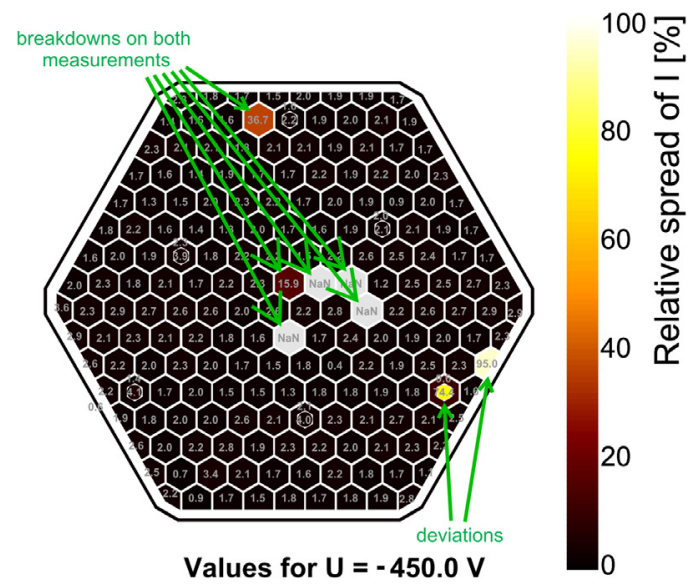

Fig. 9. Cell current deviations between backside and frontside biasing of a $200 \mu \mathrm{m}$ thick sensor. Values are given in \% (Eq. (1)) at $-450 \mathrm{~V}$.

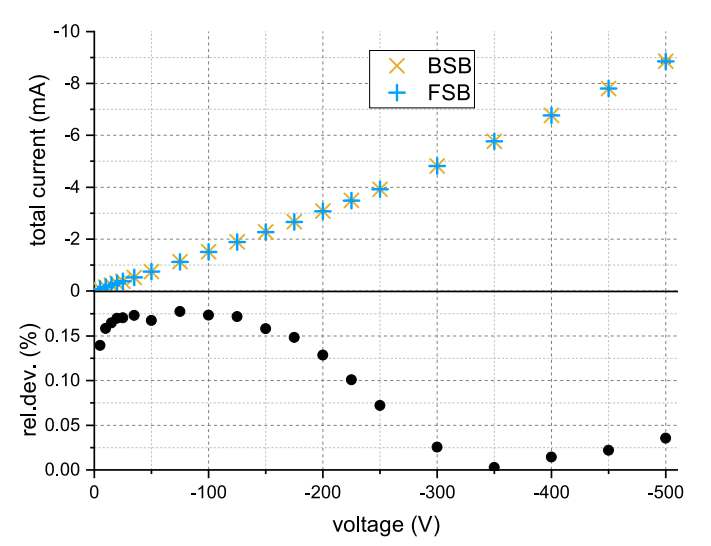

Fig. 10. Total current as a function of the bias voltage, comparison between backsideand frontside biasing schemes of a $200 \mu \mathrm{m}$ thick sensor. Relative deviations are given by Eq. (1).

contact for sensor biasing instead. This method is referred as "frontside biasing" [14].

To verify this on the $8^{\prime \prime}$ prototypes, we tested frontside biasing on an unirradiated, 192-channel $200 \mu \mathrm{m}$ thick sensor and compared frontside biasing cell currents to backside biasing cell currents. Fig. 9 shows the relative spread $(R)$ as derived in Eq. (1), between front side bias current
$I_{\mathrm{FSB}}$ and back side bias current $I_{\mathrm{BSB}}$ for each cell.

$R(\%):=\left|\frac{I_{\mathrm{BSB}}}{a}-1\right| * 100 \%$ and $a=\frac{I_{\mathrm{BSB}}+I_{\mathrm{FSB}}}{2}$.

For most cells not in breakdown, the differences are below $2 \%$. There were two exceptions with remarkable deviations up to $95 \%$, which may have resulted in handling-induced defects (see Section 3.2) by changing to backside-biasing. Currents of cells in breakdown are not considered in this analysis.

The measured total currents deviated by less than $0.2 \%$ between frontside and backside biasing, as shown in Fig. 10. Thus, we consider the frontside biasing concept as a viable option for unirradiated sensors.

In contrast to earlier sensor designs [13], inward extensions of the edge ring as shown in right Fig. 11 with contact pads (passivation openings over metalization) have now been included. Since the HGCAL will also utilize partial sensors, we applied one edge ring extension on each long sensor edge. These structures now give the possibility to contact the edge ring via probe-cards [9], see Section 3.1. The contact area of the extensions is large enough to support two redundant pin contacts on each extension to avoid sparking in case of loss contact during the testing.

For contacting the inner guard ring, we implemented a similar concept. The main purpose of these guard ring extensions, shown in left Fig. 11, is to ease the placement of wirebonds between the guard ring and the "Hexaboard". The Hexaboard is a printed circuit board (PCB) for power supply and readout of the silicon sensor. A positive side-effect is the speedup of sensor testing because dedicated probe-card contacts using spring-loaded pins can be used instead of contacting the guard ring via separate needles. In previous designs, the guard ring was too narrow to be contacted via probe-card pins. However, $0.2036 \%$ of active sensor area gets lost due to these extensions.

\section{Conclusions and prospects}

The aforementioned backside fragility requires adaptations of the current sensor testing and handling procedures such as the necessity of protecting the backside during testing. In this paper it was shown that frontside biasing is a practical solution to bypass the problem of biasing an insulated sensor backside.

To qualify the radiation hardness of the new $8^{\prime \prime}$ process, extensive irradiation tests have to be done. Radiation changes indicators like full depletion voltage and bulk resistivity, which can be extracted from IV and CV measurements. Also, irradiation campaigns of test structures are planned which allow to investigate the radiation hardness of the pstop elements [15]. Metal-Oxide-Semiconductor (MOS) test capacitors allow measuring the oxide charges via flatband voltage, which gives information about the oxide quality [6].

It is projected to verify these results in parallel by Technology Computer-Aided Design (TCAD) simulations [13] to improve understanding the effects of the $8^{\prime \prime}$ process technology on radiation hardness.

\section{Declaration of competing interest}

The authors declare that they have no known competing financial interests or personal relationships that could have appeared to influence the work reported in this paper.

\section{Acknowledgment}

This project has received funding from the call "Forschungspartnerschaften" of the Austrian Research Promotion Agency (FFG), Austria under the grant no. 868296 . 

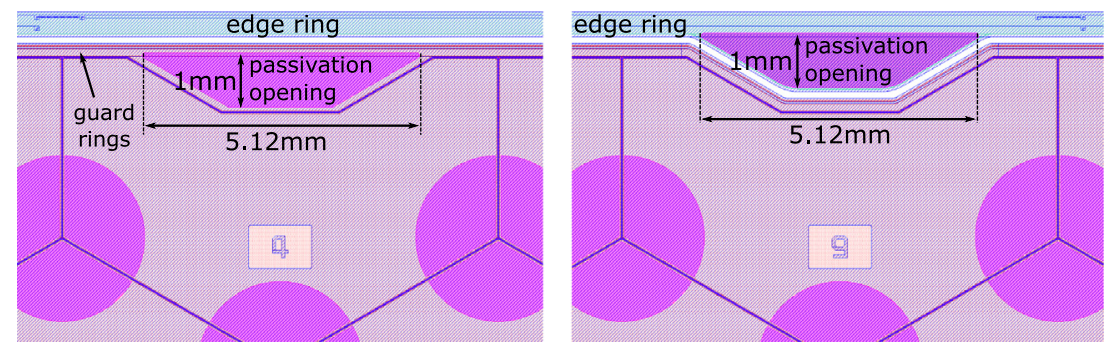

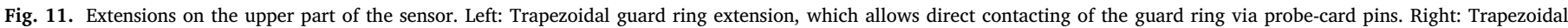
edge ring extension, which allows direct contacting of the edge ring via probe-card pins. The circular sectors are passivation openings for contacting the cell diodes.

\section{References}

[1] G. Apollinari, et al., High-Luminosity Large Hadron Collider (HL-LHC): Technical Design Report V. 0.1, Tech. rep., in: CERN Yellow Reports: Monographs, CERN, Geneva, 2017, URL https://cds.cern.ch/record/2284929.

[2] The CMS Collaboration, The CMS experiment at the CERN LHC, J. Instrum. 3 (08) (2008) S08004, http://dx.doi.org/10.1088/1748-0221/3/08/s08004.

[3] The CMS Collaboration, The Phase-2 Upgrade of the CMS Endcap Calorimeter, Tech. Rep., CERN-LHCC-2017-023. CMS-TDR-019, 2017, https://cds.cern.ch/ record/2293646.

[4] D. Barney, T. Sakuma, CMS Collaboration, Sketchup images highlighting the sub-detectors, 2017, URL https://cds.cern.ch/record/2628519.

[5] D. Barney, CMS Collaboration, Overview slide of CE with main parameters, 2019, URL https://cms-docdb.cern.ch/cgi-bin/PublicDocDB/ShowDocument? docid $=13251$.

[6] V. Hinger, Process quality control strategy for the Phase II upgrade of the CMS tracker and endcap calorimeters, Nucl. Instrum. Methods A this volume (2020).

[7] A. Affolder, P. Allport, G. Casse, Collected charge of planar silicon detectors after pion and proton irradiations up to 2.2E16neqcm-2, Nucl. Instrum. Methods A 623 (1) (2010) 177-179, http://dx.doi.org/10.1016/j.nima.2010.02.187.

[8] The CMS Tracker Collaboration, P-type silicon strip sensors for the new CMS tracker at HL-LHC, J. Instrum. 12 (06) (2017) P06018, http://dx.doi.org/10. 1088/1748-0221/12/06/p06018.
[9] E. Brondolin, et al., ARRAY: An open source, modular and probe-card based system with integrated switching matrix for characterisation of large area silicon pad sensors, Nucl. Instrum. Methods A 940 (2019) 168-173, http://dx.doi.org/ 10.1016/j.nima.2019.06.007.

[10] E. Brondolin, Silicon sensors for the CMS HGCAL upgrade: Challenges, sensor design \& electrical characterization, 2020, arXiv:2003.02461.

[11] P. Dias De Almeida, Versatile systems for characterization of large-area silicon pad sensors for highly-granular calorimetry, 2020, arXiv:2004.00851.

[12] V. Hinger, et al., Process quality control for large-scale silicon sensor productions, Nucl. Instrum. Methods A 924 (2019) 38-43, http://dx.doi.org/10.1016/j.nima. 2018.07.082.

[13] E. Pree, Development of Large Area Silicon Sensors for the High Granularity Calorimeter At CMS (Ph.D. thesis), Technische Universitat Wien, 2018, pp. 61-67, URL http://katalog.ub.tuwien.ac.at/AC15192834.

[14] M. Baselga et al., Front-side biasing of n-in-p silicon strip detectors, J. Instrum. 13 (2018) P11007, http://dx.doi.org/10.1088/1748-0221/13/11/P11007.

[15] F. Moscatelli, A. Morozzi, D. Passeri, S. Mattiazzo, G.-F.D. Betta, T. Bergauer, M. Dragicevic, V. Hinger, G. Bilei, Measurements and simulations of surface radiation damage effects on IFX and HPK test structures, Nucl. Instrum. Methods A 958 (2020) 162794, http://dx.doi.org/10.1016/j.nima.2019.162794, Proceedings of the Vienna Conference on Instrumentation 2019. 